coloproctology 2015 $37: 239$

DOI 10.1007/s00053-015-0025-8

Online publiziert: 19. Juni 2015

๑) Springer-Verlag Berlin Heidelberg 2015

\section{F. Aigner}

Charité Universitätsmedizin Berlin, Campus Virchow-Klinikum, Klinik für Allgemein-, Viszeral- und

Transplantationschirurgie, Berlin, Deutschland

\title{
Funktionalität in der kolorektalen Chirurgie
}

Die folgenden vier unter dem Leitthema „Funktionalität in der kolorektalen Chirurgie“ zusammengefassten Artikel sollen einen Überblick über dieses zunehmend an Interesse gewinnende Thema des Funktionserhalts nach kolorektalen und im Speziellen nach onkologischen Eingriffen bieten. Nach Einhaltung der geforderten Radikalitätsprinzipien bei der totalen mesorektalen Exzision (TME) haben die präoperative Erfassung der Funktion der Beckenorgane und des Beckenbodens sowie deren intraoperative Erhaltung und schließlich auch die Evaluation des postoperativen funktionellen Outcomes im Sinne der Qualitätssicherung und -kontrolle höchste Priorität. Hauptproblem in der Erfüllung dieser Vorgaben ist die nach wie vor unzureichende Aufklärung unserer Patienten, vor allem was die mögliche Beeinträchtigung der Stuhl- und Blasenentleerungsstörung sowie der Sexualfunktion betrifft [1]. Dies zu berücksichtigen hat sich die neugegründete gleichnamige Arbeitsgruppe „Funktionalität in der kolorektalen Chirurgie" der Chirurgischen Arbeitsgemeinschaft Coloproktologie (CACP) der Deutschen Gesellschaft für Allgemein- und Viszeralchirurgie (DGAV) zu einem ihrer Ziele gesetzt und widmet sich daher in den folgenden Beiträgen der komplexen pelvinen Anatomie, dem nervenschonenden Zugang bei der laparoskopischen Technik, die nicht zuletzt durch die jüngst erschienene COLOR-II-Studie [2] der offenen Technik in puncto funktionellem und offensichtlich auch onkologischem Outcome im distalen Rektumdrittel überlegen ist, und der Einstufung des anterioren Resektionssyndroms durch einen neuen und einfach anzuwendenden Score [3]. Abgerundet wird dieses Leitthema durch Vorstellung eines transanalen Zuganges zur TME (TAMIS-TME), der vor allem bei anatomischen Limitationen wie dem engen, adipösen, männlichen Becken und tief und vorne sitzendem Rektumkarzinom aufgrund der besseren Übersicht einen Sphinktererhalt unter Berücksichtigung der onkologischen Radikalitätsprinzipien ermöglicht.

\section{Korrespondenzadresse}

\section{PD Dr. F. Aigner}

Charité Universitätsmedizin Berlin, Campus Virchow-Klinikum, Klinik für Allgemein-, Viszeral-und Transplantationschirurgie Augustenburger Platz 1, 13353 Berlin, Deutschland

felix.aigner@charite.de

Interessenkonflikt. F. Aigner gibt an, dass kein Interessenkonflikt besteht.

\section{Literatur}

1. Scheer AS, O'Connor AM, Chan BP, Moloo H, Poulin EC, Mamazza J, Auer RC, Boushey RP (2012)
The myth of informed consent in rectal cancer surgery: what do patients retain? Dis Colon Rectum 55(9):970-975

2. Bonjer $\mathrm{HJ}$, Deijen $\mathrm{CL}$, Abis GA, Cuesta $\mathrm{MA}$, van der Pas $\mathrm{MH}$, de Lange-de Klerk ES, Lacy AM, Bemelman WA, Andersson J, Angenete E, Rosenberg J, Fuerst A, Haglind E (2015) COLOR II Study Group. A randomized trial of laparoscopic versus open surgery for rectal cancer. N Engl J Med 372(14):1324-1332

3. Juul T, Ahlberg M, Biondo S, Emmertsen KJ, Espin E, Jimenez LM, Matzel KE, Palmer G, Sauermann A, Trenti L, Zhang W, Laurberg S, Christensen P (2014) International validation of the low anterior resection syndrome score. Ann Surg 259(4):728-734 